\title{
Value of repeating amylase levels in abdominal pain
}

\author{
This article was published in the following Dove Press journal: \\ Open Access Surgery \\ 20 August 2010 \\ Number of times this article has been viewed
}

Rajaraman Durai' Alexandras Uzkalnis²

'Specialist registrar, ${ }^{2}$ Consultant surgeon, Department of Surgery, University Hospital Lewisham, London, SEI 3 6LH, UK
Correspondence: Rajaraman Durai Department of Surgery, University Hospital Lewisham, London, SEI3 6LH, UK

Email dr_durai@yahoo.com
Serum amylase and lipase are commonly used tests for diagnosing pancreatitis. Serum amylase level is usually high in the early days of pancreatitis which subsequently decreases. Serum lipase is not routinely available in every hospital. We report an unusual case of pancreatitis where amylase was normal initially then it began to rise.

A 71-year-old lady was admitted with an acute onset of epigastric/right upper quadrant pain without any radiation. She was also vomiting and constipated for three days. Her past medical history included open cholecystectomy, myocardial infarction, emphysema, incisional hernia repair related to cholecystectomy scar, type 2 diabetes, and hysterectomy. On examination, she was apyrexial, with normal vital signs. Clinical examination showed morbid obesity, some asymmetrical bulge in right upper quadrant above the cholecystectomy scar without cough impulse and tenderness in the epigastrium and right hypochondrium. Chest and abdominal X-rays were within the normal limits. Her initial blood tests showed an amylase of $56 \mathrm{U} / \mathrm{L}$, obstructive-type jaundice, and the remaining blood tests were within the normal range. Arterial blood gas did not show any acidosis. Her pain deteriorated. A computed tomography scan was organized, which excluded any recurrent hernia via cholecystectomy scar. A magnetic resonance cholangiopancreatography excluded any obstruction in the common bile duct. There was a diagnostic dilemma even after two scans investigating the cause of her abdominal pain. Bloods tests were repeated the following day which showed an amylase of $500 \mathrm{U} / \mathrm{L}$ and elevated urea from dehydration. She scored two on Ranson's criteria and was treated for pancreatitis. Her condition improved with analgesia and supportive treatment.

As a rule, amylase should be checked in all abdominal pain. A normal amylase does not exclude pancreatitis. Amylase is secreted by salivary gland and pancreas. The enzyme is excreted in the urine. Therefore amylase levels usually will come back to normal in three or four days after development of pancreatitis. This case is unusual because amylase was normal initially and increased after 48 hours. Sometimes it may be difficult to explain the cause of abdominal pain, particularly in an obese individual with numerous co-morbidities. Repeat amylase helped us in this case and it also avoided any unnecessary laparotomy. 